\title{
Ageism and suicide prevention
}

Diego de Leo AO, DSc, Emeritus Professor of Psychiatry, Griffith University, Brisbane

Suicide in old age is often considered to be the result of a rational decision. Frailty, dependence on others, loss of partner and loneliness are often seen as reasons that might explain many instances of suicide. Similarly, suicide can be interpreted as a legitimate "exit" in case of dramatic changes in social status and role. Ageistic views tend to consider depression as a normal feature of the aging process, and advanced age as the antecedent of an anticipated, definitive ending. Accumulation of physical illnesses and disabilities, life events, and losses can be perceived as the "right cause" to step out of this stage of life. This interpretation - the ultimate corollary of senectus ipsa morbus - overlaps with the idea of the elderly as being overwhelmed by their emotions, and seeing suicide as the only escape from an unbearable condition.

There is no doubt that depression is an important risk factor for suicidal behaviour even at very advanced age; ${ }^{1}$ however, it is possible that the its role has been overestimated or perhaps generalized more than necessary by making it the scapegoat of any situation related to suicide. In fact, excessive simplification of problems, where everything is attributable to depression, can induce a rigid prescriptive approach: literally so, as this often limits treatment to the recommendation of an antidepressant drug. Hence the appreciation of the multifactorial nature of an individual's crisis becomes too narrow. From the perspective of suicide prevention, a careful and prudent approach is always preferable to considering life eventsand reactions such as suicide - as inevitable. Such a passive approach might particularly characterise the relationship between mental health care professionals and patients of very advanced age, where stressors of physical and non-physical natures readily aggregate, multiplying their impact. ${ }^{2}$

In fact, the role of non-medical risk factors can be particularly important in the very old. Physical and emotional isolation, relocation, forced institutionalisation, lack of expectations for any improvement, and family discord are some of the problems that may represent major challenges for individuals already experiencing the consequences of somatic pathologies. ${ }^{3}$ In these cases, the role of physicians as interceptors of suicide trajectories is particularly difficult: it requires scientific knowledge, but also — or above all — the qualities of human understanding that not all doctors make available to their patients. Elderly patients often perceive doctors' hesitation and indecision; they have lived for long time and survived many difficult moments; they are expert in humans and human things; even if with some ambivalence (the one that led them to the medical consultation), they have decided to end their lives and have a precise plan in mind. ${ }^{4}$

This train of thoughts seems to characterize especially suicidal males of advanced age. It is not so much depression as a devastating combination of physical impairments, loneliness, or social invisibility. These men used to have a role, a sense of usefulness and an active presence in society: now they feel their life counts for nothing; it is tiring to carry on (taedium vitae); they think they are a burden to society and their family members (if any) ${ }^{2}$. Above all, they are convinced that their life would never change for the better. Some of them would like to be helped, but they are not used to ask for it. ${ }^{5}$ In terms of asking for help, women do better than men; indeed, despite greater longevity and exposure to a larger number 
of pathologies than men, women generally have better retention of their social network, more capacity for adaptation, greater self-sufficiency in daily activities, and are more often engaged in the care for children and grandchildren. ${ }^{6}$ Men also appear less likely than women to adhere to therapies when initiated. ${ }^{7}$ The cultural script of masculinity has promoted the suppression of emotions, discouraging men from showing sadness, sorrow, or weeping, and then inhibiting them by seeking interpersonal support in times of difficulty. ${ }^{8}$

What to do, then? While mental health care remains a pillar of suicide prevention in late life, the range of interventions should be expanded by paying more attention to the many socioenvironmental conditions that are relevant to this stage of life. Community programs that promote a sense of worthiness and belonging should be strongly encouraged in order to preserve personal identity and social integration. Loneliness must be counteracted in its many aspects and with more vigour than it has been done so far. The fight against stigma and ageistic views - still deeply rooted even among health workers $\mathbf{9 , 1 0}$ - must be carried out with greater determination. Active promotion of a culture of resilience and adaptation to different phases of life and the changes imposed by them should constitute the founding basis of all efforts aimed at promoting a successful aging process.

\section{The author does not have any conflict of interest}

\section{References}

1. De Leo D, Draper B, Snowdon J, Kolves K (2013). Suicides in older adults: A casecontrol psychological autopsy study in Australia. Journal of Psychiatric Research, 47: 980-988.

2. De Leo D, Arnautovska U (2016). Prevention and treatment of suicidality in older adults. In R O'Connor \& J Pirkis (eds), International Handbook of Suicide Prevention. Research, Policy and Practice. Wiley Blackwell, Chichester, 323-345.

3. O'Dwyer, S.T., De Leo, D. (2016). Older adults and suicide. In D Wasserman (ed), Suicide: Un Unnecessary Death. Oxford University Press, Oxford, 215-227.

4. De Leo, D. (2010). Turning Points. Australian Academic Press, Brisbane.

5. Galdas, P.M., Cheater, F. \& Marshall, P. (2005). Men and help-seeking behaviour: literature review. Journal of Advanced Nursing, 49, 616-623.

6. Canetto, S.S. (1995). Elderly women and suicidal behavior. In S.S. Canetto \& D. Lester (Eds.), Women and suicidal behaviour. New York: Springer Publishing Company, Inc.

7. Vörös, V, Osváth, P. \& Fekete, S. (2004). Gender differences in suicidal behaviour. Neuropsychopharmacology, Hungary, 6, 65-71.

8. Cochran, S.V. \& Rabinowitz, F.E. (2003). Gender-sensitive recommendations for assessment and treatment of depression in men. Professional Psychology, 2, 132-140.

9. Schulze, B. (2007). Stigma and mental health professionals: A review of the evidence on an intricate relationship. International Review of Psychiatry, 19, 137-155. 
10. Sao Jose', J.M.S., Amado, C.A.F., Ilinca, S., Buttigieg, S.C., Taghizadeh Larsson, A. (2017). Ageism in health care: A systematic review of operational definitions and inductive conceptualizations. Gerontologist, May 16. doi: 10.1093/geront/gnx020. [Epub ahead of print] 